\title{
Investigations on genetic diversity and relationships among Channa species using AFLP-capillary electrophoresis
}

\author{
AIGUO ZHOU ${ }^{1,3}$, SHAOLIN XIE ${ }^{1}$, CHAONAN ZHANG ${ }^{1}$, ZHENLU WANG ${ }^{1}$, CHAO WANG ${ }^{1}$, \\ YONGYONG FENG ${ }^{1}$, YANFENG CHEN ${ }^{2}$ AND JIXING ZOU ${ }^{1}$ \\ ${ }^{1}$ College of Marine Sciences, South China Agricultural University, Guangzhou - 510 642, Guangdong, China \\ ${ }^{2}$ School of Life Science and Engineering, Foshan University, Foshan - 528 231, Guangdong, China \\ ${ }^{3}$ Qingyuan North River Fishery Science Institute, Qingyuan - 511 510, Guangdong, China \\ e-mail: zoujixing@scau.edu.cn
}

\begin{abstract}
The genetic diversity and relationships among eight species of Channa were investigated using amplified fragment length polymorphism (AFLP)-capillary electrophoresis. Ten EcoR I/Mse I primer pairs were selected out of thirty eight and were used for further AFLP analysis. The total amplified bands, consistency of bands and polymorphic bands ranged from 114 to 206, from 0 to 12 and from 114 to 196, respectively, with corresponding averages of 156.2; 4 and 152.2 respectively. The proportion of polymorphic bands ranged from 93.62 to $100 \%$, with an average of $97.44 \%$. The similarity coefficients among eight Channa species ranged from 0.5365 to 0.7875 . The dendrogram generated after unweighted pair-group method with arithmetic means (UPGMA) clustering with NTSYS software indicated that the eight Channa species could be divided into four major clusters according to the genetic similarity coefficient of 0.58. Channa striata and Channa micropeltes were clustered into a separate branch that was far from the other six species and Channa argus kimurai (Shih) was closest to Channa argus. These results could be useful for conservation as well as for guiding research on captive breeding of Channa species.
\end{abstract}

Keywords: AFLP, Amplified fragment length polymorphism, Capillary electrophoresis, Channa species, Genetic diversity, Intergeneric relationships

\section{Introduction}

Snakehead (Perciformes: Channidae) are widely distributed in China (Cheng and Zheng, 1987; Courtenay and Williams, 2004), having high economic value and regarded as good food fish owing to their nutritional, medicinal and pharmaceutical properties (Liuetal., 2000). The classification of some species of Channidae is still controversial. For example, Channa argus kimurai shares very similar morphology and overlapping distribution with Channa argus. It is named for the white colour of its entire body and has only been found in the Jialing River in Sichuan. According to the earliest documentation, $C$. argus has three subspecies: C. argus argus (Cantor, 1842); O. argus warpachowskii Berg, 1909; and O. argus kimurai Shih, 1936 (Shih, 1936; Nichols, 1943; Nikolski and Gao, 1960). Wang et al. $(1992 ; 1993)$ suggested that O. argus kimurai should not be regarded as a subspecies of $C$. argus; rather, they concluded that it is an albino mutant of $C$. argus, based on their investigations on morphological characteristics, principal component analysis, lactate dehydrogenase and esterase isozymes. The study of $16 \mathrm{~S}$ and $12 \mathrm{~S}$ rRNA between two colour morphs of northern snakehead also confirmed this point (Zhou, 2017a, b).

Amplified fragment length polymorphism (AFLP) markers are considered one of the best molecular markers for population analysis because they can be assessed in a short period of time and it is widely applied to analyse fish populations. AFLP is a DNA molecular marker based on restriction fragment length polymorphism (RFLP) and random amplified polymorphic DNA (RAPD) (Zabeau and Vos, 1993; Vos et al., 1995). AFLP has been applied in germplasm identification, genetic diversity, genetic map construction, QTL location, animal genetics and breeding, disease detection and other areas (Li et al., 2003; Zhang et al., 2005; Liu et al., 2005; Yang et al., 2006; Krishnamurthy et al., 2015). Chen et al. (2009), identified seven geographic groups of Epinephelus akaara using AFLP in the East China Sea and South China Sea and the UPGMA phylogenetic tree revealed that E. akaara mainly clustered into geographic groups, providing a scientific basis for germplasm resource protection of E. akaara. The late larval and adult fish of 11 Lutjanus species were analysed using AFLP electrophoretic pattern in the 
Spratly Islands; the larvae were successfully identified and the phylogenetic and genetic relationships of Lutjanus were clearly explained, improving and complementing traditional morphological taxonomy findings (Zhang et al., 2005). The proportion of AFLP polymorphs of Cyprinus carpio and Ctenopharyngodon idella were 6.7 and $58.9 \%$, respectively, indicating a distant phylogenetic relationship between them. Notably, simple sequence repeat (SSR) markers showed the same results (David et al., 2011). Capillary electrophoresis (CE), also known as high-performance CE (HPCE), is a new liquid phase separation technique that employs capillary action for the separation channel and high-voltage direct current electric field as the driving force. Electrophoresis results can be statistically analysed, effectively reducing the analysis time by eliminating complex steps such as gel destaining, immobilisation, washing, silver staining, developing and fixing while greatly improving resolution. Therefore, in the present study, AFLP-capillary electrophoresis was applied to evaluate the genetic diversity and relationships among different species under the genus Channa. The study also attempted to elucidate the molecular evidence for the classification between two colour morphs of northern snakehead.

\section{Materials and methods}

\section{Sample collection and DNA extraction}

Fin clippings and muscle tissue samples were collected and immediately soaked in $95 \%$ ethanol and then stored at $-20^{\circ} \mathrm{C}$. Total genomic DNA was extracted from the caudal fin using a standard extraction kit (DNeasy tissue kit, Baitaike Biotech Co. Ltd., China), DNA concentration was measured using Amersham Biosciences Ultrospec 2100 spectrophotometer and electrophoresed on $1 \%$ agarose gels and stored at $-20^{\circ} \mathrm{C}$. Basic information of sampling sites and size of Channa species used for the study are given in Table 1.

\section{AFLP analysis}

One hundred nanogram of DNA was digested with double restriction enzyme (EcoR I/Mse I) (New England Biolabs Co., Ltd., Beijing) and were ligated to two adapters (EcoR I- adapter/ Mse I- adapter) (Table 2) with T4 DNA ligase (New England Biolabs Co., Ltd., Beijing). Digestion and ligation reactions were carried out simultaneously. The reaction was carried out using a $20 \mu$ reaction volume, containing $2 \mu$ l of $10 \times$ AFLP digest-ligation buffer, $1.8 \mu \mathrm{l}$ AFLP digest-ligation enzyme mix, $1 \mu \mathrm{l}(10 \mu \mathrm{m})$ EcoR I adaptor, $1 \mu \mathrm{l}(10 \mu \mathrm{m})$ Mse I adaptor, $5 \mu \mathrm{l}$ DNA template and up to $20 \mathrm{ul}$ of double distilled water. The reaction conditions were $5 \mathrm{~h}$ at $25^{\circ} \mathrm{C}$ and electrophoresed on $1 \%$ agarose gel. One microliter of the ligated product was used for pre-amplification by PCR with adapter-homologous primers with one selective nucleotide. The PCR amplification profile was as follows: $3 \mathrm{~min}$ at $94^{\circ} \mathrm{C}$, followed by 20 cycles of $30 \mathrm{~s}$ at $94^{\circ} \mathrm{C}, 30 \mathrm{~s}$ at $50^{\circ} \mathrm{C}$ and $1 \mathrm{~min}$ at $72^{\circ} \mathrm{C}$.

The pre-amplification product was diluted 20-folds and was subjected to selective amplification using primers with three selective nucleotides and ten pairs of selective AFLP primers (Table 2) were chosen. The selective amplification reaction was performed with touchdown PCR begun at $95^{\circ} \mathrm{C}$ for $5 \mathrm{~min}$, followed by 12 cycles of $35 \mathrm{~s}$ at $95^{\circ} \mathrm{C}, 35 \mathrm{~s}$ at $65^{\circ} \mathrm{C}$ (decreasing $0.7^{\circ} \mathrm{C}$ each cycle) for $30 \mathrm{~s}, 1 \mathrm{~min}$ at $72^{\circ} \mathrm{C}$. The last reaction was performed at $94^{\circ} \mathrm{C}$ for $30 \mathrm{~s}, 56^{\circ} \mathrm{C}$ for $30 \mathrm{~s} ; 72^{\circ} \mathrm{C}$ for $1 \mathrm{~min}$ with 23 cycles and final extension of $72^{\circ} \mathrm{C}$ for $5 \mathrm{~min}$.

\section{Capillary electrophoresis}

Formamide and molecular weight internal standards were mixed in the ratio of 100:1. Fifteen microliter was taken with 1 ul amplified product (diluted 10-folds) and was sent to Bio-ulab Biotech Co., Ltd. for high

Table 1. Basic information of sampling sites and size (n) of the eight Channa species

\begin{tabular}{|c|c|c|c|c|}
\hline Species & Location & GPS coordinates & Altitude (m) & Year of collection \\
\hline C. argus kimurai & Neijiang City, Sichuan Province, China & $\begin{array}{l}29^{\circ} 34^{\prime} 14.644^{\prime \prime} \mathrm{N} \\
105^{\circ} 4^{\prime} 1.95^{\prime \prime} \mathrm{E}\end{array}$ & 332 & 2014 \\
\hline C. argus & San Jiaozhen, Zhongshan City, Guangdong & $22^{\circ} 39^{\prime} 14.311^{\prime} \mathrm{N}$ & 1 & 2014 \\
\hline C. maculata & Province, China & $113^{\circ} 25^{\prime} 43.95^{\prime \prime} \mathrm{E}$ & & 2015 \\
\hline 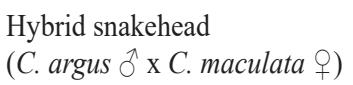 & $\begin{array}{l}\text { Shiban Cun, Qingyuan City, Guangdong } \\
\text { Province, China }\end{array}$ & $\begin{array}{l}23^{\circ} 44^{\prime} 33.06^{\prime \prime} \mathrm{N} \\
113^{\circ} 4^{\prime} 28.84^{\prime \prime} \mathrm{E}\end{array}$ & 16 & 2015 \\
\hline $\begin{array}{l}\text { C. asiatica } \\
\text { C. micropeltes }\end{array}$ & Guangzhou City, Guangdong Province, China & $\begin{array}{l}23^{\circ} 5^{\prime} 3.60^{\prime \prime} \mathrm{N} \\
113^{\circ} 14^{\prime} 46.24^{\prime \prime} \mathrm{E}\end{array}$ & 3 & $\begin{array}{l}2015 \\
2015\end{array}$ \\
\hline C. lucius & Hanoi City, Vietnam & $\begin{array}{l}21^{\circ} 1^{\prime} 41.45^{\prime \prime} \mathrm{N} \\
105^{\circ} 51^{\prime} 48.94 ” \mathrm{E}\end{array}$ & 164 & 2014 \\
\hline C. striata & Vientiane City, Laos & $\begin{array}{l}17^{\circ} 57^{\prime} 52.27^{\prime} \mathrm{N} \\
102^{\circ} 35^{\prime} 17.97^{\prime} \mathrm{E}\end{array}$ & 167 & 2014 \\
\hline
\end{tabular}


Table 2. Adapters and primer sequences used in the analysis of AFLP-capillary electrophoresis

\begin{tabular}{llll}
\hline Adapters and primers & Sequence $\left(5^{\prime}\right.$-3' $\left.^{\prime}\right)$ & Primers & Sequence $\left(5^{\prime}\right.$-3 $\left.^{\prime}\right)$ \\
\hline Eco R adapter 1 & CTCGTAGACTGCGTACC & Mse I adapter 1 & GACGATGAGTCCTGAG \\
Eco I adapter 2 & AATTGGTACGCAGTCTAC & Mse I adapter 2 & TACTCAGGACTCAT \\
Pre-Pe & GACTGCGTACCAATTC & Pre-Pm & GATGAGTCCTGAGTAA \\
E-AAC & GACTGCGTACCAATTCAAC & M-CCA & GATGAGTCCTGAGTAACCA \\
E-AAG & GACTGCGTACCAATTCAAG & M-CGA & GATGAGTCCTGAGTAACGA \\
E-ACT & GACTGCGTACCAATTCACT & M-CGC & GATGAGTCCTGAGTAACGC \\
E-AGC & GACTGCGTACCAATTCAGC & M-CTA & GATGAGTCCTGAGTAACTA \\
E-ATC & GACTGCGTACCAATTCATC & M-CTC & GATGAGTCCTGAGTAACTC \\
E-ATG & GACTGCGTACCAATTCATG & M-CTG & GATGAGTCCTGAGTAACTG \\
E-ATT & GACTGCGTACCAATTCATT & M-CTT & GATGAGTCCTGAGTAACTT \\
E-CAC & GACTGCGTACCAATTCCAC & M-GAC & GATGAGTCCTGAGTAAGAC \\
E-CAG & GACTGCGTACCAATTCCAG & M-GAG & GATGAGTCCTGAGTAAGAG \\
E-CAT & GACTGCGTACCAATTCCAT & M-GAT & GATGAGTCCTGAGTAAGAT \\
\hline
\end{tabular}

performance capillary electrophoresis employing ABI 3730XL.

\section{Data analysis}

For each individual, the DNA fingerprints were scored by apparatus inspection (ABI 3730) and the files were analysed with Genemarker V2.20. Polymorphic DNA bands ranging from 40-620 bp were scored as present (1) or absent (0). The genetic distance between each Channa species was calculated using the NTSYSpc 2.11 software package (Rohlf, 1997). The dendrogram for the eight Channa species were constructed using the unweighted pair-group method with arithmetic means (UPGMA) in MEGA 4.1 based on the genetic distance (Tamura et al., 2007).

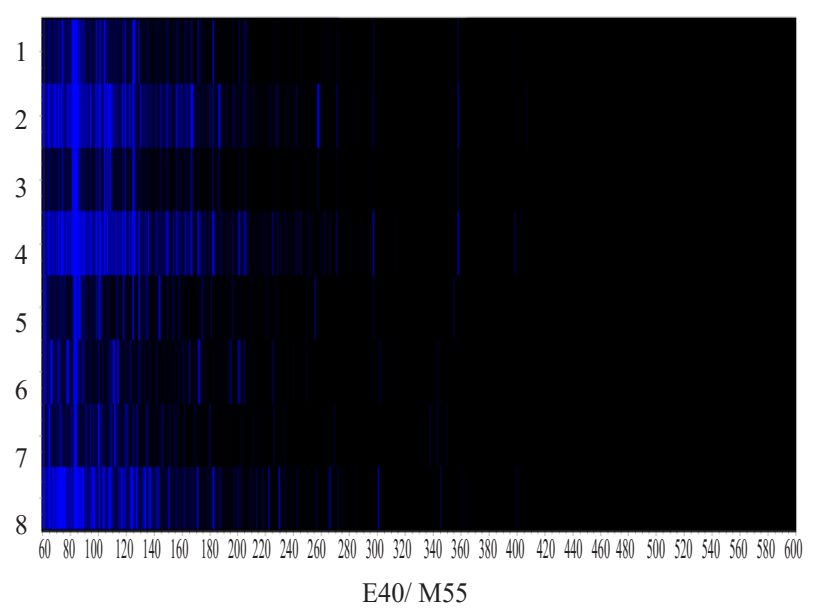

(a)

\section{Results}

Population genetic variation of Channa species

The proportions of polymorphic loci provide a good measure of genetic variations among the eight Channa species. A total of 1562 loci were detected by the ten AFLP primer pairs, of which 1522 (97.44\%) were polymorphic (Table 3 ). The total loci scored per primer pair ranged from 114 to 206 loci with average of 156.2. The average number of polymorphic loci scored per primer was 152.2 loci and ranged from 114 to 196 loci (Table 3). The proportion of the polymorphic loci ranged from 65.96 to $81.97 \%$ with an average of $97.44 \%$ (Table 3). The number of amplification loci and the

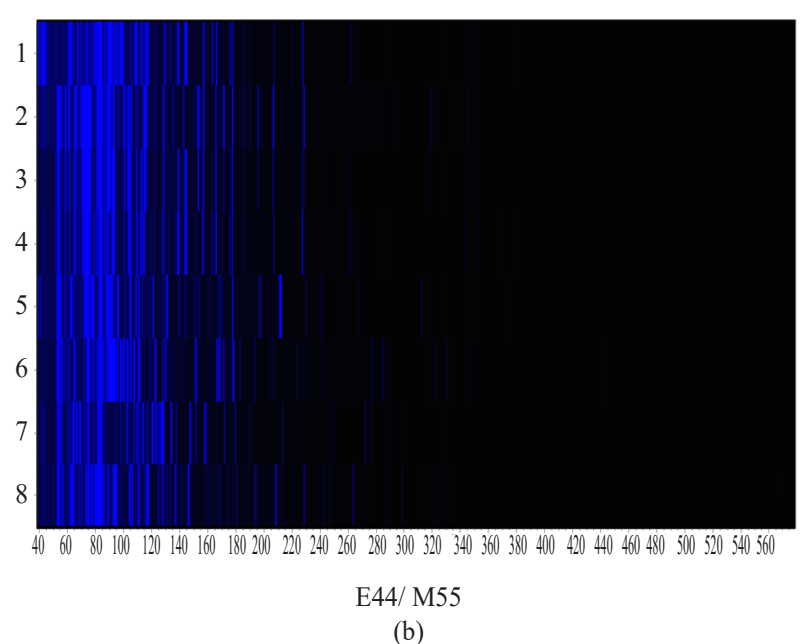

(b)

Fig. 1. AFLP high performance capillary electrophoresis gel patterns generated by (a) the lowest primer combination (E44/M59) and (b) the highest primer combination (E40/M55).

1. C. argus, 2. C. maculata, 3. Hybrid snakehead, 4. C. argus kimurai, 5. C. asiatica, 6. C. striata, 7. C. micropeltes, 8. C. lucius 
Table 3. The amplification results per AFLP primer pair of eight Channa species

\begin{tabular}{lllll}
\hline Primer pair & Number of total loci & Number of consistent loci & Number of polymorphic loci & Proportion of polymorphic loci (\%) \\
\hline E32/M61 & 132 & 4 & 128 & 96.97 \\
E33/M60 & 142 & 4 & 138 & 97.18 \\
E38/M66 & 188 & 12 & 176 & 93.62 \\
E40/M55 & 206 & 10 & 196 & 95.15 \\
E44/M59 & 114 & 0 & 114 & 100.00 \\
E45/M62 & 192 & 2 & 190 & 98.96 \\
E46/M56 & 131 & 2 & 129 & 98.47 \\
E48/M51 & 197 & 3 & 194 & 98.48 \\
E49/M65 & 124 & 1 & 123 & 99.19 \\
E50/M64 & 136 & 2 & 134 & 98.53 \\
Total & 1562 & 40 & 1522 & 97.66 \\
Mean & 156.2 & 4 & 152.2 &
\end{tabular}

proportion of polymorphic loci were both large, which suggests that the AFLP marker provides abundant genetic information and it was a perfect genetic marker for population genetic analysis. AFLP-high performance capillary electrophoresis gel patterns generated by ten primers are depicted in Fig. 1. Only the highest (Fig. 1a) and lowest (Fig. 1b) number of primer combination are presented.

Population structure and differentiation among eight Channa species

A total of 10 pairs of AFLP primer combinations were used to detect genetic variation of pooled genomic DNA from eight Channa species (Table 4). The genetic distance and similarity among the eight Channa species ranged from 0.3425 to 1.0392 and from 0.5365 to 0.7875 . Based on the genetic similarity coefficients, the UPGMA dendrogram displayed four major clusters. The genetic similarity coefficients between $C$. argus and $C$. argus kimurai was the greatest $(0.7875)$ and then clustered with hybrid snakehead and C. maculata. C. asiatica and C. lucius formed a basal cluster, the relationship between C. striata and C. micropeltes was considerably far from each other and from other Channa species (Fig. 2).

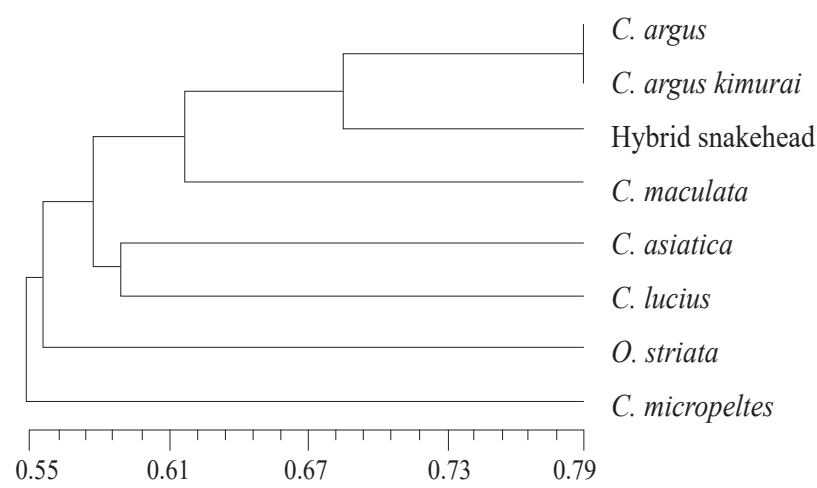

Fig. 2. Phylogenetic tree constructed for eight Channa species using UPGMA method

\section{Discussion}

AFLP-capillary electrophoresis is a highly efficient molecular marker technique. It can amplify more than 200 sites with a pair of primers and detect site differences as small as $1 \mathrm{bp}$, which makes it easier to detect subtle differences among materials. Moreover, it reduces the error caused by manual operation, because it employs an efficient automation system that ensures standardised analysis of amplified samples and improves data reliability

Table 4. Genetic distance (above diagonal) and similarity coefficients (below diagonal) among eight Channa species based on AFLP data

\begin{tabular}{lllllllll}
\hline Species & Channa argus & C. maculata & Hybrid snakehead & C. argus kimurai & C. asiatica & C. striata & C. micropeltes & C. lucius \\
\hline C. argus & $* * * *$ & 0.7712 & 0.6246 & 0.3425 & 0.9075 & 0.8690 & 0.9421 & 0.9541 \\
C. maculata & 0.5698 & $* * * *$ & 0.5126 & 0.7144 & 0.8638 & 0.8575 & 0.8217 & 0.9240 \\
Hybrid snakehead & 0.6620 & 0.6863 & $* * * *$ & 0.5229 & 0.8209 & 0.8762 & 0.9707 & 0.9487 \\
C. argus kimurai & 0.7875 & 0.5992 & 0.7106 & $* * * *$ & 0.9074 & 0.8834 & 0.9151 & 0.9411 \\
C. asiatica & 0.5768 & 0.5589 & 0.6152 & 0.5871 & $* * * *$ & 0.9143 & 0.9253 & 0.9939 \\
O. striata & 0.5621 & 0.5365 & 0.5723 & 0.5672 & 0.5717 & $* * *$ & 0.8733 & 1.0209 \\
C. micropeltes & 0.5410 & 0.5499 & 0.5461 & 0.5589 & 0.5698 & 0.5589 & $* * * *$ & 1.0392 \\
C. lucius & 0.5762 & 0.5519 & 0.5915 & 0.5903 & 0.5922 & 0.5557 & 0.5525 & $* * * *$ \\
\hline
\end{tabular}


(O'Hanlon and Peakall, 2000). In addition, high-quality DNA is required for establishing an AFLP reaction system and double digestion of genomic DNA and adapter binding are important factors in developing an AFLP system. Therefore, the key to success in the present study was the strict requirements for binding, pre-amplification and selective amplification.

In the selective amplification using 10 combinations of EcoR I and Mse I primers, each pair could generate 1562 clear sites (distributed between 60 and $600 \mathrm{bp}$ ). The average number of total loci scored per primer pair was 156.2. The primer combination E40/M55 amplified a maximum of 206 sites, while the lowest number was for the primer combination of E44/M59, which amplified 114. Each primer combination could generate highly polymorphic sites, the highest reached $100 \%$ and the average was $97.44 \%$. Most teleost studies using AFLP focus on genetic diversity among different populations of the same species. A previous study of seven E. akaara groups found large differences in genetic diversity among different geographical groups (Chen et al., 2009). An AFLP analysis of population genetic diversity of Trachidermus fasciatus revealed a low level of genetic diversity between two regional populations (Xu et al., 2008). A comparative study of genetic diversity based on RAPD and AFLP markers reported a significantly higher polymorphism detection efficiency of AFLP markers compared to RAPD markers (Yang and Yu, 2006). Previous studies have successfully identified Cyprinus carpio, Acceder oxyrinchus and their hybrids, indicating that the technology can be extended to identify other similar organisms (Congiu et al., 2011). Many researchers have employed AFLP to assess genetic relationships among plant species and successfully identified a high proportion of polymorphisms. AFLP can obtain more information for a given species and can effectively assess genetic diversity among species; therefore, it can play an important role in improving and complementing the traditional morphological taxonomy studies (Liu et al., 2005; Zhang et al., 2005).

C. argus kimurai shares very similar morphology and overlapping distribution with $C$. argus. There has been considerable controversy regarding its classification. According to the earliest documentation, they belong to two different subspecies. Some researchers have suggested that $C$. argus kimurai cannot be regarded as a subspecies of $C$. argus; rather, they consider it an albino mutant (Shih, 1936; Wang et al., 1992; 1993; Zhou 2017a, b).

The phylogenetic tree constructed for the eight Channa species based on UPGMA cluster analysis shows that $C$. argus kimurai and $C$. argus have the highest genetic similarity coefficient (0.7875) (Fig. 2). This further demonstrates that from the molecular biology perspective, C. argus kimurai may be an albino mutant rather than a subspecies of $C$. argus. These findings provide important molecular clues for correcting the classification of $C$. argus kimurai under the genus Channa.

The hybrid snakehead had a relatively small genetic distance from the parents, but the three species could be distinctly separated in the clustering tree (Fig. 2). This also demonstrates that AFLP-capillary electrophoresis is applicable to germplasm analysis for cross-breeding, especially when the parents and their offsprings have similar morphologies. Therefore this molecular marker could be effectively used for distinguishing the genetic effects of hybridisation between wild species, as well as for germplasm identification.

\section{Acknowledgements}

We gratefully thank Dr. Zhuo X. L. for the assistance provided in fish sampling and Dr. Liang R. S. and Cui K. for their assistance in data analysis. This study was supported by the Science and Technology Planning Project of Guangdong Province (2017A020225035; 2016A020210141); Produce-learn-research Project of Guangdong Province (2011B090400270); Fund Fostering Talents for Young Scholars of South China Agricultural University (201707N025); Talent introduction special funds of South China Agricultural University and Scientific Research Staring Foundation for Young Scholars of College of Marine Sciences. All of the procedures and animal handling were performed in accordance with the guide for the Chinese Association for Laboratory Animal Sciences. Approval of the study was obtained from the Animal Ethics Committee of South China Agricultural University. We also express our appreciation to our anonymous reviewers for providing valuable comments on the manuscript.

\section{References}

Chen, S. P., Hu, X. L. and Liu, T. 2009. AFLP analysis of seven geographical populations of Epinephelus akaara. Acta Scientiarum Naturalium Universitat is SunYatseni, 48: 56-61.

Cheng, Q. T. and Zheng, B. S. 1987. Systematic search of fish in China, Science Press, Beijing, 456 pp.

Congiu, L., Dupanloup, I., Patamello, T., Fontana, F., Rossi, R., Arlati, G. and Zane, L. 2011. Identification of interspecific hybrids by amplified fragment length polymorphism: the case of sturgeon. Mol. Ecol., 10: 2355-2359.

Courtenay, W. R. J. and Williams, J. D. 2004. Snakeheads (Pisces, Channidae): A biological synopsis and risk assessment, US Department of the Interior, US Geological Survey, Circular, $143 \mathrm{pp}$. 
David, L., Fang, J. G., Rajasekaran, P., Hillel, J. and Lavi, U. 2011. Polymorphism in ornamental and common carp strains (Cyprinus carpio L.) as revealed by AFLP analysis and a new set of microsatellite markers. Mol. Genet. Genomics, 266: $353-362$.

Krishnamurthy, S. L., Prashanth, Y., Rao, A. M., Reddy, K. M. and Ramachandra, R. 2015. Assessment of AFLP marker based genetic diversity in chilli (Capsicum annuum L. \& $C$. baccatum L.). Indian J. Biotechnol., 14: 49-54.

Li, Y., Byrne, K., Miggiano, E., Whan, V., Moore, S., Keys, S., Crocos, P., Preston, N. and Lehnert, S. 2003. Genetic mapping of the kuruma prawn, Penaeus japonicus using AFLP markers. Aquaculture, 219: 143-156.

Liu, B. Q., Dong, W. Q., Wang, Y. J., Zhu, S. H. and Wu, W. X. 2005. Identification of germ plasm in Pseudosciaena crocea Tai-Chu Race by AFLP. Acta Hydrobiol. Sin., 29: 413-416.

Liu, J., Cui, Y. and Liu, J. 2000. Resting metabolism and heat increment of feeding in mandarin fish (Siniperca chuatsi) and Chinese snakehead (C. argus). Comp. Biochem. Physiol., 127:131-138.

Liu, Y., Chen, S., Li, B., Wang, Z. J. and Liu, Z. J. 2005. Analysis of genetic variation in selected stocks of hatchery flounder, Paralichthys olivaceus, using AFLP markers. Biochem. Syst. Ecol., 33: 993-1005.

Nichols, J. T. 1943. The freshwater fishes of China, Natural History of Central Asia, vol. 9. American Museum of Natural History, New York, USA.

Nikolski and Gao, Y. 1960. The fish of Heilongjiang River, Science Press, Beijing, p. 394-399.

O'Hanlon, P. C. and Peakall, R. 2000. A simple method for the detection of size homoplasy among amplified fragment length polymorphism fragments. Mol. Ecol., 9: 815-816.

Rohlf, F. J. 1997. NTSYS-pc version 2.0. Exeter Software, Applied Biostatistics Incorporated.

Shih, H. J. 1936. Notes on the labyrinth fishes of China. Bull. Fan. Mem. Lnst. Biol. Peiping (Zool.), 7: 81-82.

Tamura, K., Dudley, J., Nei, M. and Kumar, S. 2007. MEGA4: Molecular Evolutionary Genetics Analysis (MEGA) software version 4.0. Mol. Biol. Evol., 24: 1596-1599.
Vos, P., Hoger, R. and Blecker, M. 1995. AFLP: a new technique for DNA fingerpri-nting. Nucleic Acids Res., 23: 4407-4414.

Wang, J. X., Liao, Z. G., Zhang, Z. G. and Zhao, X. F. 1993. Principal components analysis of the Ophicephalus argus complex (Pisces: Channidae). J.ournal of Southwest China Normal University (Natural Science), 2: 168-172.

Wang, J. X., Zhao, X. F., Zhou, C. W. and Liao, Z. G. 1992. Comparative studies on the Ophicephalus argus and $O$. argus kimurai, with the systematics of Ophicephalus argus. Trans. Ocean. Limnol., 2: 51-57.

Xu, J. R., Han, X. L., Li, N., Yu, J. F., Xu, P. and Bao, Z. M. 2008. Analysis of genetic diversity in roughskin sculpin Trachidermus fasciatus by AFLP markers. J. Dalian Fish. Univ., 23: 437-441.

Yang, D. and Yu, L. N. 2006. Comparison of genetic diversity of Oreochromis niloticus by AFLP and RAPD Analysis. Acta Agriculturae Jiangxi, 18:1-4.

Yang, S., Ye, X., Lu, M. X., Huang, Z. H. and Bai, J. J. 2006. AFLP analysis of two species of tilapia, Oreochromis mossambicus and O. hornorum. Periodical of Ocean University of China, 36: 937-940.

Zabeau, M. and Vos, P. 1993. Selective restriction fragment amplification: a general method for DNA fingerprinting, European Patent Application: European, 0543858A1 [P/ OL].

Zhang, J. B., Huang, L. M. and Chen, Z. R. 2005. Studies on the larval identification and taxonomy of Lutjanus by AFLP technology. Acta Oceanol. Gica Sin., 27: 165-171.

Zhou, A. G., Wang, C., Jiang, W. Z., Li, Z. G., Chen, Y. F., Xie, S. L., Luo, J. Z. and Zou, J. X. 2017a. Genetic comparison of two colour morphs of northern snakehead (Channa argus) and genetic relatedness among the family Chnannidae. Mitochondrial DNA Part A, 28(6): 971-973.

Zhou, A. G., Xie, S. L., Wang, W. Z., Fan, L. F., Wang, C., Ye, Q., Chen, Y. F. and Zou, J. X. 2017b. Molecular evidence of two colour morphs of northern snakehead (Channa argus) based on mitochondrial 12S rRNA. Mitochondrial DNA Part B, 2(1): $283-286$ 